Wright State University

CORE Scholar

Physics Faculty Publications

Physics

8-1-2005

\title{
Introduction and Recovery of Point Defects in Electron-Irradiated $\mathrm{ZnO}$
}

F. Tuomisto

K. Saarinen

David C. Look

Wright State University - Main Campus, david.look@wright.edu

Gary C. Farlow

Wright State University - Main Campus, gary.farlow@wright.edu

Follow this and additional works at: https://corescholar.libraries.wright.edu/physics

Part of the Physics Commons

\section{Repository Citation}

Tuomisto, F., Saarinen, K., Look, D. C., \& Farlow, G. C. (2005). Introduction and Recovery of Point Defects in Electron-Irradiated ZnO. Physical Review B, 72 (8), 85206.

https://corescholar.libraries.wright.edu/physics/208

This Article is brought to you for free and open access by the Physics at CORE Scholar. It has been accepted for inclusion in Physics Faculty Publications by an authorized administrator of CORE Scholar. For more information, please contact library-corescholar@wright.edu. 


\title{
Introduction and recovery of point defects in electron-irradiated $\mathrm{ZnO}$
}

\author{
F. Tuomisto* and K. Saarinen \\ Laboratory of Physics, Helsinki University of Technology, P.O. Box 1100, 02015 TKK, Finland \\ D. C. Look \\ Semiconductor Research Center, Wright State University, Dayton, Ohio 45435, USA \\ and Materials and Manufacturing Directorate, Air Force Research Laboratory, Wright-Patterson AFB, Ohio 45433, USA \\ G. C. Farlow \\ Physics Department, Wright State University, Dayton, Ohio 45435, USA \\ (Received 6 April 2005; revised manuscript received 13 June 2005; published 3 August 2005)
}

\begin{abstract}
We have used positron annihilation spectroscopy to study the introduction and recovery of point defects in electron-irradiated $n$-type $\mathrm{ZnO}$. The irradiation $\left(E_{\mathrm{el}}=2 \mathrm{MeV}\right.$, fluence $\left.6 \times 10^{17} \mathrm{~cm}^{-2}\right)$ was performed at room temperature, and isochronal annealings were performed from 300 to $600 \mathrm{~K}$. In addition, monochromatic illumination of the samples during low-temperature positron measurements was used in identification of the defects. We distinguish two kinds of vacancy defects: the $\mathrm{Zn}$ and $\mathrm{O}$ vacancies, which are either isolated or belong to defect complexes. In addition, we observe negative-ion-type defects, which are attributed to $\mathrm{O}$ interstitials or $\mathrm{O}$ antisites. The $\mathrm{Zn}$ vacancies and negative ions act as compensating centers and are introduced at a concentration $\left[V_{\mathrm{Zn}}\right] \simeq c_{\text {ion }} \simeq 2 \times 10^{16} \mathrm{~cm}^{-3}$. The $\mathrm{O}$ vacancies are introduced at a 10-times-larger concentration $\left[V_{\mathrm{O}}\right] \simeq 3 \times 10^{17} \mathrm{~cm}^{-3}$ and are suggested to be isolated. The $\mathrm{O}$ vacancies are observed as neutral at low temperatures, and an ionization energy of $100 \mathrm{meV}$ could be fitted with the help of temperature-dependent Hall data, thus indicating their deep donor character. The irradiation-induced defects fully recover after the annealing at $600 \mathrm{~K}$, in good agreement with electrical measurements. The $\mathrm{Zn}$ vacancies recover in two separate stages, indicating that the $\mathrm{Zn}$ vacancies are parts of two different defect complexes. The $\mathrm{O}$ vacancies anneal simultaneously with the $\mathrm{Zn}$ vacancies at the later stage, with an activation energy of $E_{V, O}^{m}=1.8 \pm 0.1 \mathrm{eV}$. The negative ions anneal out between the two annealing stages of the vacancies.
\end{abstract}

DOI: 10.1103/PhysRevB.72.085206

PACS number(s): 61.72.Ji, 61.82.Fk, 78.20.Ek, 78.70.Bj

\section{INTRODUCTION}

Interest in zinc oxide $(\mathrm{ZnO})$ has grown during the recent years due to progress in crystal growth ${ }^{1}$ and its unique optical and electrical properties. $\mathrm{ZnO}$ has a direct band gap of about $3.4 \mathrm{eV}$ at $2 \mathrm{~K}$, similarly to gallium nitride $(\mathrm{GaN})$, for which it can be thought of as an alternative for use in optoelectronic devices. The recent reports on successful $p$-type doping of $\mathrm{ZnO}$ (Refs. 2-4) have led to even higher expectations about the material's potential applications in electronics. In addition, when doped with manganese, $\mathrm{ZnO}$ has been reported to exhibit ferromagnetic behavior above room temperature, ${ }^{5}$ making it a prospective material for spintronics applications.

Point defects have an important effect on the electronic and optoelectronic properties of semiconductor materials. Both their identification and quantification are necessary in order to understand the microscopic processes leading to the specific properties. Point defects are created in semiconductors during growth, where their formation is governed by thermodynamics and growth kinetics. They can be introduced in concentrations much larger than those given by the thermodynamic equilibrium by means of irradiation of the material-e.g., by electrons. The study of the formation of point defects under these nonequilibrium conditions gives information on the basic physical properties of the semiconductor material.

Defects introduced by electron and neutron irradiation in $\mathrm{ZnO}$ have been studied by electron paramagnetic resonance
(EPR) in the past ${ }^{6-11}$ and also quite recently. ${ }^{12,13}$ Signals from both $\mathrm{Zn}$ and $\mathrm{O}$ interstitials ${ }^{12}$ and from $\mathrm{Zn}$ vacancies ${ }^{6-9}$ have been observed only at temperatures well below the room temperature-i.e., at 4-90 K. The disparition of the signals has been reported to occur at several stages between $110 \mathrm{~K}$ and $280 \mathrm{~K}$. Only the $\mathrm{O}$ vacancies have been observed with EPR at room temperature. . $^{10,11,13}$ The photoluminescence spectrum of $\mathrm{ZnO}$ typically exhibits a green luminescence (GL) band similar to the yellow luminescence in GaN. ${ }^{14}$ Both the Zn (Refs. 14,15) and O (Refs. 16-18) vacancies have been proposed to be the defect responsible for this GL band. However, the conclusive identification is still lacking.

Both the optical and electrical properties of $\mathrm{ZnO}$ are much more resistant than those of $\mathrm{GaN}$ to deterioration caused by room-temperature electron and proton irradiation, ${ }^{19-21}$ which makes it potentially useful for applications in highirradiation environments. The apparent radiation hardness remains when the irradiation is performed at a low temperature of $130 \mathrm{~K},{ }^{22}$ indicating that the primary irradiation-induced compensating defects are mobile already at those temperatures. The microscopic origin of the radiation hardness of $\mathrm{ZnO}$ is not, however, well understood.

In this work we investigate the introduction and thermal recovery of the point defects created in $\mathrm{ZnO}$ by roomtemperature irradiation with $2-\mathrm{MeV}$ electrons. To do this we apply positron annihilation spectroscopy, which is sensitive to defects with open volume. Positrons get trapped at va- 
cancy defects, which changes their annihilation characteristics. The positron lifetime reflects the open volume of the defect. On the other hand, the Doppler broadening of the $511-\mathrm{keV}$ annihilation radiation gives information about the electron momentum distribution at the annihilation site and is sensitive to the atomic environment of the vacancy. The combination of these techniques can thus be used to identify both the open volume and the sublattice of the defect in compound semiconductors.

We observe three kinds of point defects produced by the electron irradiation $\left(E_{\mathrm{el}}=2 \mathrm{MeV}\right.$, fluence $\left.6 \times 10^{17} \mathrm{~cm}^{-2}\right)$ : the $\mathrm{Zn}$ and $\mathrm{O}$ vacancies or complexes involving the vacancies and negative-ion-type defects, which are attributed to $\mathrm{O}$ interstitials or $\mathrm{O}$ antisites. The $\mathrm{Zn}$ vacancies and negative-iontype defects act as compensating centers and are introduced at a concentration $\left[V_{\mathrm{Zn}}\right] \simeq c_{\mathrm{st}} \simeq 2 \times 10^{16} \mathrm{~cm}^{-3}$. The $\mathrm{O}$ vacancies are introduced at a 10-times-larger concentration $\left[V_{\mathrm{O}}\right]$ $\simeq 3 \times 10^{17} \mathrm{~cm}^{-3}$. The $\mathrm{O}$ vacancies are observed as neutral at low temperatures, and an ionization energy of $100 \mathrm{meV}$ could be fitted with the help of temperature-dependent Hall data, thus indicating their deep donor character. By shining monochromatic light on the samples during the positron annihilation measurements at low temperature, we find that both the $\mathrm{Zn}$ vacancies and the negative-ion-type defects have ionization levels close to $2.3 \mathrm{eV}$. Thus both defects are possibly involved in the green luminescence exhibited by ZnO. ${ }^{14,17}$ The irradiation-induced defects fully recover after the annealing at $600 \mathrm{~K}$, in good agreement with electrical measurements. ${ }^{19}$ The $\mathrm{Zn}$ vacancies anneal out of the material in two stages with fitted activation energies $E_{A 1}^{V, Z n} \simeq 1.3 \mathrm{eV}$ and $E_{A 2}^{V, Z n} \simeq 1.8 \mathrm{eV}$, indicating that the $\mathrm{Zn}$ vacancies are parts of two different defect complexes. The $\mathrm{O}$ vacancies anneal simultaneously with the $\mathrm{Zn}$ vacancies at the later stage with fitted activation energy $E_{A}^{V, O} \simeq 1.8 \mathrm{eV}$. The $\mathrm{O}$ vacancies are suggested to be isolated, and hence the activation energy can be interpreted as the migration barrier of the $\mathrm{O}$ vacancy. The negative-ion-type defects anneal out between the two annealing stages of the $\mathrm{Zn}$ and $\mathrm{O}$ vacancies.

\section{METHOD}

\section{A. Experimental details}

The positron lifetime experiments were performed in nominally undoped single-crystal $\mathrm{ZnO}$ samples grown by the seeded vapor phase technique. Two samples were irradiated with $2-\mathrm{MeV}$ electrons to a fluence $6 \times 10^{17} \mathrm{~cm}^{-3}$. The irradiations were performed with a current density of $2 \mathrm{~A} / \mathrm{cm}^{2}$ at room temperature, in a vacuum of $1-5 \times 10^{-6}$ torr. Both the as-grown and irradiated samples were $n$ type (in asgrown $N_{e} \simeq 1 \times 10^{17} \mathrm{~cm}^{-3}$ and in irradiated $N_{e} \simeq 3$ $\times 10^{15} \mathrm{~cm}^{-3}$ ), most likely due to residual $\mathrm{H}$ and $\mathrm{Al}$. After irradiation, the samples were mounted to a closed-cycle liquid helium cryostat for positron measurements as a function of isochronal annealing between 300 and $600 \mathrm{~K}$. The 30-min annealings were performed in situ in the positron measurement cryostat in a vacuum of $10^{-6}$ mbar. The positron lifetime measurements were performed at 20,80, 150, and 300 $\mathrm{K}$.
The positron measurement cryostat was equipped with a possibility to shine monochromatic light on the samples during lifetime measurements. These measurements were performed under monochromatic illumination with sub-bandgap $(h \nu=0.7-3.1 \mathrm{eV})$ light. The photon flux was kept constant during each measurement, but at high photon energies only a somewhat lower flux was obtained due to limitations in the intensity of the light source (halogen lamp). Photon fluxes between $10^{14}$ and $10^{15} \mathrm{~cm}^{-2} \mathrm{~s}^{-1}$ were used. Additionally, two 540-nm green lasers were used to produce a photon flux $6 \times 10^{15} \mathrm{~cm}^{-2} \mathrm{~s}^{-1}$ at photon energy $h \nu$ $=2.3 \mathrm{eV}$. All the measurements of the photoexcitation of the defects were performed at $20 \mathrm{~K}$.

The positron lifetimes were measured with a conventional fast-fast coincidence spectrometer with a time resolution of 250 ps. ${ }^{23}$ Two identical sample pieces were sandwiched with a $20-\mu \mathrm{Ci}$ positron source $\left({ }^{22} \mathrm{Na}\right.$ deposited on $1.5-\mu \mathrm{m} \mathrm{Al}$ foil). Typically $2.5 \times 10^{6}$ and $5 \times 10^{6}$ annihilation events were collected in each positron lifetime spectrum in the annealing experiments and in the photoexcitation experiments, respectively. The lifetime spectrum $n(t)=\Sigma_{i} I_{i} \exp \left(-t / \tau_{i}\right)$ was analyzed as the sum of exponential decay components convoluted with the Gaussian resolution function of the spectrometer, after subtracting the constant background and annihilations in the source material (200 ps, 2.0\%; $400 \mathrm{ps,}, 5.8 \%$; $1500 \mathrm{ps}, 0.06 \%)$. The positron in state $i$ annihilates with a lifetime $\tau_{i}$ and an intensity $I_{i}$. The state in question can be the delocalized state in the lattice or the localized state at a vacancy defect. The increase of the average lifetime $\tau_{\text {ave }}$ $=\sum_{i} I_{i} \tau_{i}$ above the bulk lattice lifetime $\tau_{B}$ shows that vacancy defects are present in the material. This parameter is insensitive to the decomposition procedure, and even as small a change as $1 \mathrm{ps}$ in its value can be reliably measured.

In the case of one type of vacancy defect with specific lifetime $\tau_{V}$, the decomposition of the lifetime spectrum into two components $\tau_{1}$ and $\tau_{2}$ is straightforward to interpret. The second lifetime component $\tau_{2}=\tau_{V}$ gives directly the vacancyspecific lifetime and the first lifetime component is $\tau_{1}=\left(\tau_{B}^{-1}\right.$ $\left.+\kappa_{V}\right)^{-1}<\tau_{B}$, where $\tau_{B}$ is the positron lifetime in the delocalized state in the lattice and $\kappa_{V}$ the positron trapping rate into the vacancy defects.

The Doppler broadening of the annihilation radiation was measured simultaneously with the positron lifetime using a Ge detector with an energy resolution of $1.3 \mathrm{keV}$. The conventional valence $\left(S\right.$, low momenta: $\left.\left|p_{z}\right|<3 \times 10^{-3} m_{0} c\right)$ and core (W, high momenta: $10 \times 10^{-3} m_{0} c<\left|p_{z}\right|<30 \times 10^{-3} m_{0} c$ ) annihilation parameters were determined from the measured data after background subtraction as the fractions of annihilations in the corresponding momentum ranges. The parameters are presented here as relative to the parameters specific to the annihilation from the delocalized state in the perfect $\mathrm{ZnO}$ lattice.

\section{B. Data analysis}

\section{Positron trapping at defects}

The temperature dependence of the positron annihilation parameters $\left(\tau_{\text {ave }}, S\right.$, and $\left.W\right)$ is analyzed with the model of trapping and escape rates of positrons, explained in detail in 
earlier works. ${ }^{23-26}$ In this model, the trapping coefficient $\mu_{V}$ to a neutral vacancy is independent of temperature and to a negatively charged vacancy it varies as $T^{-0.5}$. The trapping rate of positrons into the vacancies (concentration $c_{V}$ ) is $\kappa_{V}$ $=\mu_{V} c_{V}$. Positrons can get trapped also at hydrogenlike Rydberg states surrounding negative-ion-type defects (shallow traps for positrons). The positron trapping rate at the Rydberg state $\mu_{R}$ varies also as $T^{-0.5}$, which is the result predicted by theory for the transition from a free state to a bound state in a Coulomb potential. ${ }^{26}$ The thermal escape rate from the Rydberg state can be written as

$$
\delta_{\mathrm{st}}=\mu_{\mathrm{R}}\left(\frac{m_{+} k_{B} T}{2 \pi \hbar^{2}}\right)^{3 / 2} \exp \left(-E_{b, \mathrm{st}} / k_{B} T\right)
$$

where $\mu_{R}$ is the positron trapping coefficient to the lowest hydrogenlike Rydberg state, $E_{b, \mathrm{st}}$ is the positron binding energy of the lowest Rydberg state (typically $<0.1 \mathrm{eV}$ ), and $m_{+} \simeq m_{0}$ is the effective mass of the positron. In principle, positrons can also escape from the Rydberg states around negatively charged vacancies, but we assume that the transition from the Rydberg state to the ground state in the vacancy is fast enough so that this effect can be neglected. This is supported by the results obtained previously in both $\mathrm{ZnO}$ (Ref. 21) and GaN (Ref. 27). An effective trapping rate of the shallow traps can thus be defined as

$$
\kappa_{\mathrm{st}}^{\mathrm{eff}}=\frac{\kappa_{\mathrm{st}}}{1+\delta_{\mathrm{st}} / \lambda_{\mathrm{st}}},
$$

where $\lambda_{\mathrm{st}} \simeq \lambda_{B}$ is the annihilation rate of positrons trapped at the Rydberg state, which coincides with the annihilation rate $\lambda_{B}$ from the delocalized state in the bulk lattice, and $\kappa_{\mathrm{st}}$ $=\mu_{R} c_{\mathrm{st}}$ is directly related to the concentration of the negative ions.

The temperature dependence of the positron data can also arise from a change in the charge state of the positron trapping defect (vacancy or negative ion) due to the dependence of the Fermi level $E_{F}$ on temperature. Then a vacancy that is neutral (as is expected for, e.g., the $\mathrm{O}$ vacancy) at low temperatures can be ionized and become positive and thus repulsive to positrons when the temperature is raised. Then the trapping rate can change even if the trapping coefficient itself is constant as a function of temperature. The ratio of neutral to all (neutral and positive) vacancies can be written as

$$
\frac{\left[V^{0}\right]}{\left[V^{0}\right]+\left[V^{+}\right]}=\left[1+g^{-1} \exp \left(\frac{E_{i}-E_{F}(T)}{k_{B} T}\right)\right]^{-1},
$$

where $E_{i}$ is the ionization energy of the neutral vacancy and $g$ the ratio of internal degeneracies of the two charge states.

In addition, the effective trapping of positrons into neutral defects can depend on temperature if the binding energy is low enough. A typical positron binding energy to a vacancy is $\sim 1 \mathrm{eV}$, and thus in the above equations the escape rate of the positrons from the ground state in the vacancy is assumed to be very low compared to the positron lifetime. However, in the case of the $\mathrm{N}$ vacancy in $\mathrm{GaN}$ or the $\mathrm{O}$ vacancy in $\mathrm{ZnO}$, where the missing atom is very small compared to the neighboring atoms, the binding energy can be of the same order of magnitude as the Rydberg state of a nega- tive ion. In such a case, the temperature dependence of the thermal detrapping causes a temperature dependence in the effective trapping rate to the neutral vacancy.

\section{Kinetic trapping model}

The decomposition of the lifetime spectra into several lifetime components gives the possibility to determine experimentally the fractions of positrons annihilating in various states. The average lifetime can be written as ${ }^{23}$

$$
\tau_{\mathrm{ave}}=\eta_{B} \tau_{B}+\sum_{j} \eta_{D, j} \tau_{D, j},
$$

where $\eta_{B}$ and $\tau_{B}$ are the annihilation fraction and positron lifetime in the free state in the lattice, and $\eta_{D, j}$ and $\tau_{D, j}$ are the corresponding values in bound states at the defect $D_{j}$. The annihilation fractions are related to the trapping rates through

$$
\eta_{B}=\frac{\lambda_{B}}{\lambda_{B}+\sum_{j} \kappa_{D, j}^{\mathrm{eff}}}, \quad \eta_{D, j}=\frac{\kappa_{D, j}^{\mathrm{eff}}}{\lambda_{B}+\sum_{j^{\prime}} \kappa_{D, j^{\prime}}^{\mathrm{eff}}} .
$$

Equation (4) can be fitted to the $\tau_{\text {ave }}$ vs $T$ data, using the trapping rates and the possible binding energies to the Rydberg states as fitting parameters. Another possibility is to write the trapping rate $\kappa_{D, j}^{\text {eff }}$ to a defect $D_{j}$ as a function of the experimental parameters:

$$
\kappa_{D, j}^{\mathrm{eff}}=\lambda_{B} \frac{\tau_{\mathrm{ave}}-\tau_{B}}{\tau_{D, j}-\tau_{\mathrm{ave}}}-\sum_{j^{\prime} \neq j} \kappa_{D, j^{\prime}}^{\mathrm{eff}} \frac{\tau_{D, j^{\prime}}-\tau_{\mathrm{ave}}}{\tau_{D, j}-\tau_{\mathrm{ave}}} .
$$

In the case of only one type of defect, the sum in Eq. (6) vanishes and the trapping rate can be calculated directly from the experimental values of $\tau_{\mathrm{ave}}, \tau_{D, j}$, and $\tau_{B}$. If several defect types are present, the trapping rates to the other defects need to be known.

\section{IDENTIFICATION OF THE DEFECTS}

\section{A. Positron data}

The results of the positron lifetime and Doppler broadening measurements in both the as-grown and irradiated $\mathrm{ZnO}$ samples are presented as a function of measurement temperature in Fig. 1. The increase of the average positron lifetime $\tau_{\text {ave }}$ with decreasing temperature is a clear indication of the presence of negatively charged vacancy defects. In the asgrown material, the monotonous behavior of the lifetime below $300 \mathrm{~K}$ indicates that these vacancy defects are the dominant negatively charged defect. On the other hand, in the electron-irradiated samples the decrease of the positron lifetime with decreasing temperature is typical of negative-iontype defects that compete in trapping of positrons with the negative vacancies. This is observed also as the increase of the first lifetime component $\tau_{1}$, where the increased trapping in the negative-ion-type defects brings the component closer to the bulk lifetime. The Doppler broadening parameters $S$ and $W$ behave as a function of temperature similarly to the positron lifetime. 


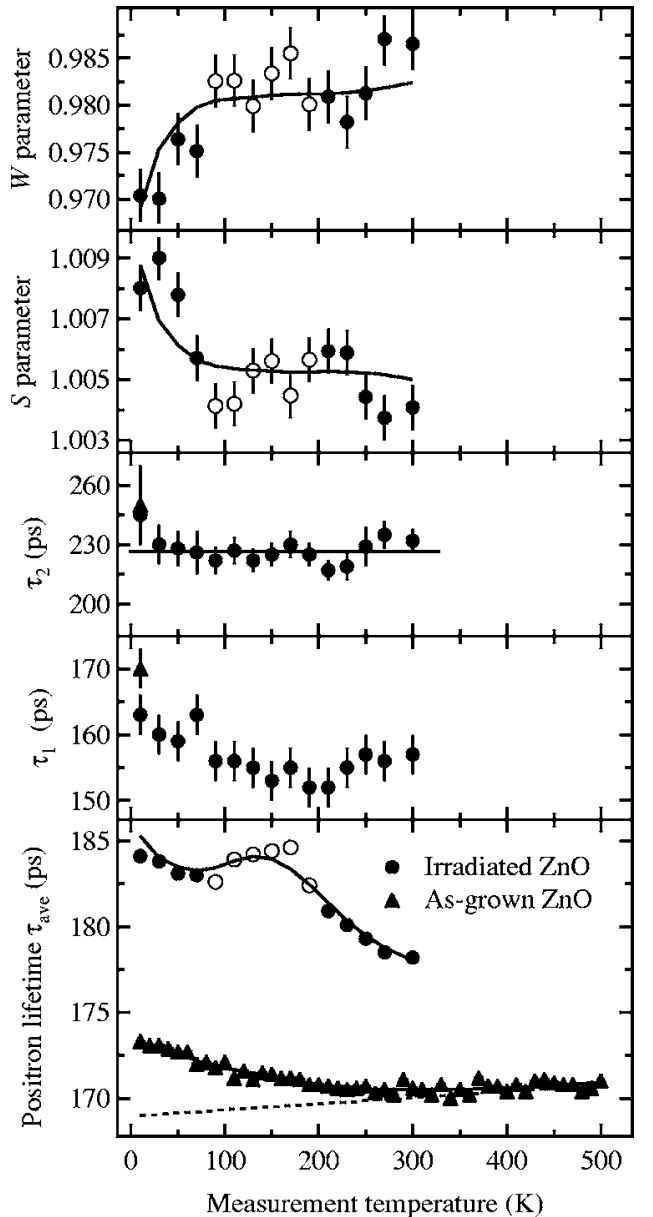

FIG. 1. The positron lifetime and Doppler broadening parameters of the as-grown and irradiated samples plotted as a function of measurement temperature. The solid curves represent the parameters obtained by fitting the temperature-dependent trapping model to the data. The dashed line shows the fitted bulk lifetime, where the temperature dependence is due to the thermal expansion of the lattice. The circles are drawn as open in the temperature range 90-190 $\mathrm{K}$, where the effect of the oxygen vacancies is most visible.

A higher lifetime component of $\tau_{V}=\tau_{2}=230 \pm 10 \mathrm{ps}$ could be extracted from the exponential lifetime spectra below 300 $\mathrm{K}$, while the data measured in the as-grown $\mathrm{ZnO}$ sample above $300 \mathrm{~K}$ were purely one-componential. The higher lifetime component is constant as a function of temperature, which implies that it represents positrons annihilating in one bound state. In our previous study, we have identified this higher lifetime component as that specific to the $\mathrm{Zn}$ vacancy and the lifetime $\tau_{B}=170 \mathrm{ps}$ to the annihilation from the delocalized state in the $\mathrm{ZnO}$ lattice at $300 \mathrm{~K} .^{21}$ Also the Doppler broadening parameters $\left(S_{D}=1.039\right.$ and $\left.W_{D}=0.87\right)$ specific to the $\mathrm{Zn}$ vacancy were determined in that work. By comparing the positron results to temperature-dependent Hall measurements, the $\mathrm{Zn}$ vacancy in the double-negativecharge state was identified as the dominant compensating defect (concentration $\left[V_{\mathrm{Zn}}\right] \simeq 2 \times 10^{15} \mathrm{~cm}^{-3}$ ) in as-grown $\mathrm{ZnO}$ and co dominant together with the so-far-unidentified negative-ion-type defect [prominent candidates are the $\mathrm{O}$ interstitial and $\mathrm{O}$ antisite, which, according to theory, are nega-

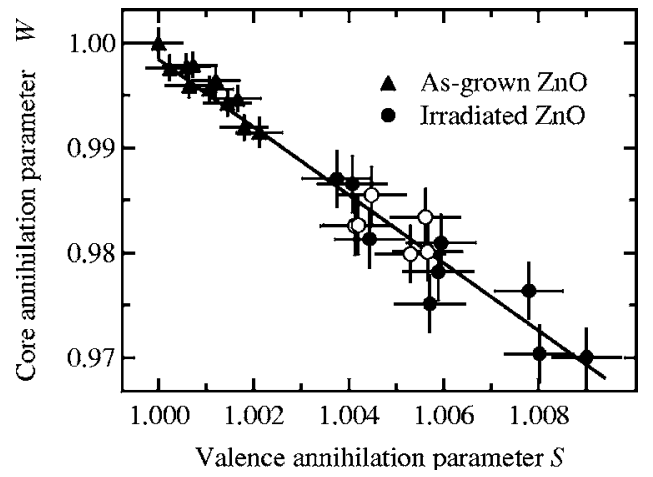

FIG. 2. The $W$ parameter plotted as a function of the $S$ parameter. The dependence is linear, indicating the presence of one type of vacancy defects. The open circles were measured at $T$ $=90-190 \mathrm{~K}$. The solid line is fitted to the data.

tive in $n$-type $\mathrm{ZnO}$ (Ref. 15)] in the irradiated material, where the concentrations of both defects were obtained as $\left[V_{\mathrm{Zn}}\right] \simeq c_{\mathrm{st}} \simeq 2 \times 10^{16} \mathrm{~cm}^{-3}$. The identification was based on both positron lifetime and Doppler broadening coincidence measurements together with comparison to theoretical positron calculations. In addition, the $\mathrm{Zn}$ vacancies were the main defect observed by positron annihilation spectroscopy.

The Doppler broadening parameters $S$ and $W$ fall on a line when plotted against each other with temperature as the running parameter (Fig. 2), which typically indicates the presence of only two distinguishable positron states (bulk and vacancy). The negative-ion-type defects do not cause deviations from the straight line, since the annihilation parameters of positrons trapped at these shallow traps coincide with the bulk parameters.

However, as can be seen in Fig. 3, the points measured at 90-190 K in the irradiated samples fall off the straight line determined by the annihilations in the bulk lattice and the $\mathrm{Zn}$ vacancy. This implies that a third positron state can be distinguished in the lifetime versus Doppler parameter data although the Doppler data itself are linear. In order to cause a deviation from the straight line, the localization to this defect needs to be strong, implying that the defect has a distinguishable open volume. The open volume of this defect cannot be very large, since, as pointed out above, the independence of temperature of the higher lifetime component $\tau_{2}$ shows no evidence of the mixing of several lifetime components. Hence, the lifetime specific to this defect needs to be sufficiently far from $\tau_{2}$ (and closer to $\tau_{1}$ ), below 200 ps. In addition, in order to produce the deviation observed in Fig. 3, the defect-specific lifetime needs to be above $\tau_{\text {ave }}$ over the whole temperature range-i.e., above 185 ps.

One additional aspect of the third type of defects is evident from the $\tau_{\text {ave }}$ vs $T$ data. The fraction of positrons annihilating as trapped at this defect is vanishing at room temperature and clearly smaller than the annihilation fractions to the $\mathrm{Zn}$ vacancies and negative-ion-type defects below $90 \mathrm{~K}$, but larger at the intermediate temperature 90-190 K. This implies that enhancement of positron trapping with decreasing temperature is larger in this defect at temperatures 190$300 \mathrm{~K}$, but saturates around $150 \mathrm{~K}$, where the $\mathrm{Zn}$ vacancies and negative-ion-type defects become more important. This 


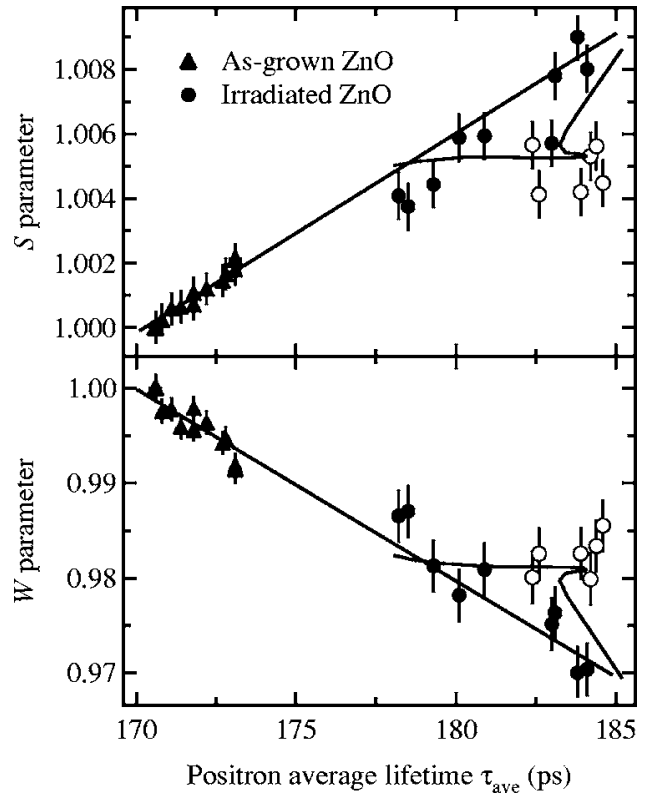

FIG. 3. The $S$ and $W$ parameters plotted as a function of $\tau_{\text {ave }}$. The parameters depend linearly on each other in the temperature range where the low-binding-energy positron traps do not contribute significantly. The open circles were measured at $T=90-190 \mathrm{~K}$. The solid lines connect the parameters of the bulk lattice to those (not shown) of the $\mathrm{Zn}$ vacancy. The solid curves are obtained from the fits to the $S$ vs $T, W$ vs $T$, and $\tau_{\text {ave }}$ vs $T$ data.

indicates that the third type of defect is neutral and the temperature dependence of the positron trapping observed at high temperatures is due to either thermal escape from the defect or a change in the charge state of the defect. Based on these considerations and especially the lifetime value of 190-200 ps, a prominent candidate for this defect is the O vacancy, which has a donor nature and would naturally have a smaller open volume than the $\mathrm{Zn}$ vacancy.

In summary, the positron data show the presence of three different defects: the negatively charged $\mathrm{Zn}$ vacancy $\left(\tau_{V, \mathrm{Zn}}\right.$ $=230 \pm 10 \mathrm{ps}$ ), the neutral (at low temperatures) $\mathrm{O}$ vacancy $\left(\tau_{V, O}=190-200 \mathrm{ps}\right)$, and the negative-ion-type defect, tentatively identified as the $\mathrm{O}$ interstitial or $\mathrm{O}$ antisite.

\section{B. Analysis}

The temperature-dependent trapping model can be used to estimate the trapping rates to the different defects from the $\tau_{\text {ave }}$ vs $T$ data in Fig. 1 using the defect-specific positron lifetimes $\tau_{V, Z \mathrm{n}}=230 \mathrm{ps}, \tau_{V, O}=195 \mathrm{ps}$, and $\tau_{\mathrm{st}}=170 \mathrm{ps}$ for the $\mathrm{Zn}$ vacancies, $\mathrm{O}$ vacancies, and negative-ion-type defects, respectively. A good first approximation can be obtained by using Eq. (6) and assuming that at $300 \mathrm{~K}$ only the $\mathrm{Zn}$ vacancies trap positrons, giving

$$
\kappa_{V, \mathrm{Zn}}=\lambda_{B} \frac{\tau_{\mathrm{ave}}-\tau_{B}}{\tau_{V, \mathrm{Zn}}-\tau_{\mathrm{ave}}} \simeq 0.2 \lambda_{B} \simeq 1.2 \times 10^{9} \mathrm{~s}^{-1} .
$$

At $30 \mathrm{~K}$ the positron data are dominated by the annihilations in the $\mathrm{Zn}$ vacancies and the negative-ion-type defects due to the fast increase in the trapping coefficient at low tempera- tures, where also $S$ and $W$ vs $\tau_{\text {ave }}$ are linear; thus, the trapping rate to the negative-ion-type defects (at $30 \mathrm{~K}$ ) can be estimated as

$$
\kappa_{\mathrm{st}}=\kappa_{V, \mathrm{Zn}} \frac{\tau_{V, \mathrm{Zn}}-\tau_{\mathrm{ave}}}{\tau_{\mathrm{ave}}-\tau_{B}}-\lambda_{B} \simeq 0.5 \lambda_{B} .
$$

Here we have used the $T^{-0.5}$ dependence of the trapping coefficient of the $\mathrm{Zn}$ vacancies (giving $\kappa_{V, \mathrm{Zn}} \simeq 0.5 \lambda_{B}$ at $30 \mathrm{~K}$ ). In addition, the escape rate from the Rydberg state of the negative ion has been neglected, since it becomes important only above $50 \mathrm{~K}$. The best estimate for the trapping rate to the $\mathrm{O}$ vacancies is obtained from the data at $100-150 \mathrm{~K}$, where the effect of the $\mathrm{O}$ vacancies is most visible. Assuming a typical value of $E_{b}=40 \mathrm{meV}$ (Ref. 28) for the Rydbergstate binding energy in addition to the $T^{-0.5}$ dependence of the trapping coefficient of the negative-ion-type defect, the (saturated) trapping rate to the $\mathrm{O}$ vacancies below $150 \mathrm{~K}$ can be estimated as

$$
\kappa_{V, \mathrm{O}}=\left(\lambda_{B}+\kappa_{\mathrm{st}}^{\mathrm{eff}}\right) \frac{\tau_{\mathrm{ave}}-\tau_{B}}{\tau_{V, \mathrm{O}}-\tau_{\mathrm{ave}}}-\kappa_{V, \mathrm{Zn}} \frac{\tau_{V, \mathrm{Zn}}-\tau_{\mathrm{ave}}}{\tau_{V, \mathrm{O}}-\tau_{\mathrm{ave}}} \simeq 0.7 \lambda_{B} .
$$

The concentrations of the defects can be estimated from the trapping rates in a straightforward matter. Assuming a trapping coefficient of $\mu_{V, \mathrm{Zn}}=3 \times 10^{15} \mathrm{~s}^{-1}$ at $300 \mathrm{~K}$ for the $\mathrm{Zn}$ vacancy as for the $\mathrm{Ga}$ vacancy in $\mathrm{GaN},{ }^{29}$ we obtain $\left[V_{\mathrm{Zn}}\right]$ $\simeq 2 \times 10^{16} \mathrm{~cm}^{-3}$. At $30 \mathrm{~K}, \kappa_{\mathrm{st}} \simeq \kappa_{V, \mathrm{Zn}}$, and assuming that the trapping coefficient of the negative-ion-type defect is similar to that of a negative vacancy, ${ }^{23,26}$ the concentrations are also similar, $c_{\mathrm{st}} \simeq 2 \times 10^{16} \mathrm{~cm}^{-3}$. These results are the same as reported previously. ${ }^{21}$ The trapping coefficient of a neutral vacancy is lower than that of a negative vacancy, ${ }^{30}$ and taking a value of $\mu_{V, O}=1 \times 10^{15} \mathrm{~s}^{-1}$ for the neutral $\mathrm{O}$ vacancy results in a concentration of $\left[V_{\mathrm{O}}\right] \simeq 3 \times 10^{17} \mathrm{~cm}^{-3}$. The concentrations of these defects are larger than the impurity concentrations reported by the manufacturer of the samples (Eagle Picher). Hence most of the irradiation-induced point defects are not complexed with the in-grown impurities.

A more detailed picture of the trapping rates to the different defects is obtained by fitting the temperature-dependent trapping model to the $\tau_{\text {ave }}$ vs $T$ data in Fig. 1. Five fitting parameters are needed in total: three absolute levels of the trapping rates and two parameters to describe the temperature dependence of the trapping to the negative-ion-type defects (Rydberg-state binding energy) and to the $\mathrm{O}$ vacancies (binding or ionization energy). Assuming that the temperature dependence of the trapping to the $\mathrm{O}$ vacancies is due to the thermal escape of positrons at high temperatures does not fit well to the data, since the changes in the trapping rate are not steep enough to reproduce the experimentally observed temperature-dependent behavior. Hence, we assign the ionization of the neutral $\mathrm{O}$ vacancy as the source of the temperature dependence of its trapping rate.

Figure 4 shows the carrier concentration and the position of the Fermi level in the irradiated $\mathrm{ZnO}$ samples obtained from temperature-dependent Hall measurements. The O vacancies start to trap positrons effectively below $200 \mathrm{~K}$, where the Fermi level is about $E_{C}-0.1 \mathrm{eV}$. Thus the ionization en- 


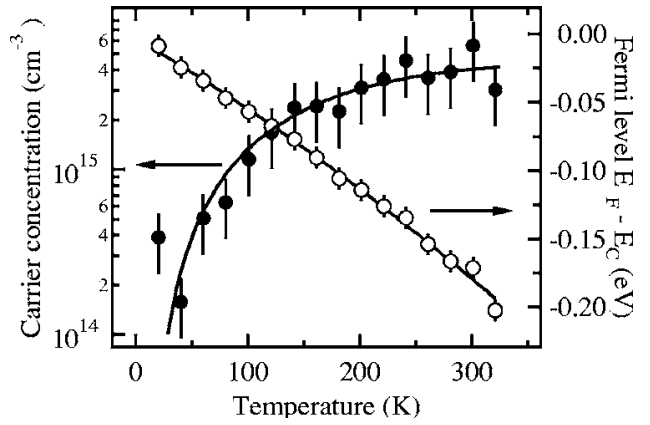

FIG. 4. The carrier concentration measured in irradiated $\mathrm{ZnO}$ as a function of temperature. The position of the Fermi energy relative to the conduction-band edge is shown. The solid curves are drawn to guide the eye.

ergy of the $\mathrm{O}$ vacancy can be expected to be around 100 $\mathrm{meV}$. Indeed, the fit results in $E_{i}=100 \pm 10 \mathrm{meV}$.

The fitted trapping rates are shown in the lower panel of Fig. 5. In addition to the ionization energy of the $\mathrm{O}$ vacancy, the binding energy to the Rydberg state of the negative-iontype defect is obtained as $E_{b}=40 \pm 10 \mathrm{meV}$, similar as observed in $\mathrm{GaN}^{28}$ The origin of temperature-dependent behavior of the positron data can be seen from the figure. The trapping rate to the $\mathrm{Zn}$ vacancies increases as $T^{-0.5}$ with decreasing temperature, while the thermal detrapping causes the trapping rate to the negative ions to vanish above $150 \mathrm{~K}$. The trapping rates to the two negative defects coincide at temperatures below $70 \mathrm{~K}$.

The trapping rate to the $\mathrm{O}$ vacancies is negligible at 300 $\mathrm{K}$, but increases rapidly with decreasing temperature. At temperatures $100-200 \mathrm{~K}$, it is significantly higher than the sum of the trapping rates to the $\mathrm{Zn}$ vacancies and the negative-ion-type defects, causing the deviation from the

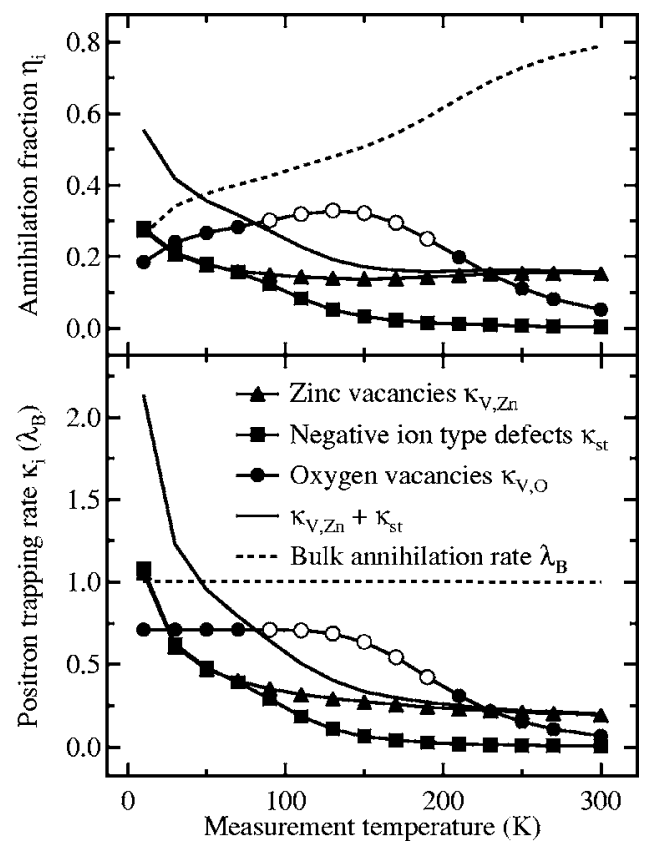

FIG. 5. The positron trapping rates and corresponding annihilation fractions of the different defects as a function of temperature. one-vacancy-type defect model (straight line) in Fig. 3. Below $100 \mathrm{~K}$, the trapping rate to the negative defects increases very rapidly and the trapping rate to the $\mathrm{O}$ vacancies has saturated to a constant value (all the $\mathrm{O}$ vacancies are neutral), making the effect of the $\mathrm{O}$ vacancies on the positron data small. The effect is seen in the positron annihilation fractions (upper panel of Fig. 5), where it can be observed that the annihilation fraction of the $\mathrm{O}$ vacancies exhibits a maximum at around $130 \mathrm{~K}$.

The trapping rates obtained from the fitting are the same as those estimated directly from the data. Thus no revisions are needed to the defect concentrations reported above. The results of the trapping rate fit can be used to obtain an estimate of the Doppler broadening parameters specific to the $\mathrm{O}$ vacancy. Fitting the same model to the $S$ vs $T$ and $W$ vs $T$ data with fixed trapping rates and using the O-vacancyspecific $S$ and $W$ parameters as fitting parameters gives $S_{V, O}=1.003$ (2) and $W_{V, O}=0.989$ (5). The negative-ionspecific parameters were taken to be those of the bulk-i.e., $S_{\mathrm{st}}=W_{\mathrm{st}}=S_{B}=W_{B}=1-$ and the parameters of the Zn vacancy were fixed at $S_{V, Z n}=1.039$ and $W_{V, Z n}=0.87 .{ }^{21}$ The fitted curves are shown in Fig. 3 and in the two upper panels in Fig. 1.

The experimentally obtained annihilation parameters specific to the $\mathrm{O}$ vacancy can be compared to those obtained from calculations. ${ }^{21}$ The calculations predict that the changes in the parameters (relative to bulk) should be small compared to those of the $\mathrm{Zn}$ vacancy, as is observed. The changes are clearly larger than predicted $\left(\tau_{V, O}^{c}-\tau_{B}^{c}=3 \mathrm{ps}\right.$ and $W_{V, \mathrm{O}}^{c}$ $=0.998)$. The calculations in the case of the $\mathrm{O}$ vacancy were performed with a theoretically predicted ${ }^{15}$ inward relaxation of neighboring atoms of approximately $8 \%$. However in early EPR measurements (at room temperature) on electronirradiated $\mathrm{ZnO}$ the isolated $\mathrm{O}$ vacancy has been observed to exhibit a substantial outward relaxation. ${ }^{11}$ Performing the same calculations as previously ${ }^{21}$ with an outward relaxation of $8 \%$ gives a lifetime difference of $\tau_{V, O}^{c}-\tau_{B}^{c}=20 \mathrm{ps}$, in reasonable agreement with the experimental lifetime. The relaxation does not affect significantly the calculated momentum distribution. The theoretical calculations also show that the dominant contribution to the Doppler broadening at high electron momenta (i.e., in the range of the $W$ parameter) comes from the $3 d$ electrons of $\mathrm{Zn}$. Hence in the case of the $\mathrm{Zn}$ vacancy the $W$ parameter decreases significantly due to the reduced annihilations with the $3 d$ electrons of $\mathrm{Zn}$, while in the case of the $\mathrm{O}$ vacancy the changes are minimal. The experimental parameters of the $\mathrm{O}$ vacancy are practically those of bulk $\mathrm{ZnO}$, which gives further support for the identification of the small open volume defects as the $\mathrm{O}$ vacancy rather than a $\mathrm{Zn}$ vacancy related complex with a substantial inward relaxation.

The introduction rate of a defect $D$ can be estimated as $\Sigma_{D}=[D] / \Phi$, where $\Phi=6 \times 10^{17} \mathrm{~cm}^{-2}$ is the irradiation fluence. With the defect concentrations $\left[V_{\mathrm{Zn}}\right] \simeq c_{\mathrm{st}} \simeq 2$ $\times 10^{16} \mathrm{~cm}^{-3}$ and $\left[V_{\mathrm{O}}\right] \simeq 3 \times 10^{17} \mathrm{~cm}^{-3}$, the corresponding introduction rates are obtained as $\Sigma_{V, \mathrm{Zn}} \simeq \Sigma_{\mathrm{st}} \simeq 0.03 \mathrm{~cm}^{-1}$ and $\Sigma_{V, O} \simeq 0.5 \mathrm{~cm}^{-1}$. The introduction rate of the $\mathrm{Zn}$ vacancies is about 30 times lower than determined for $\mathrm{Ga}$ vacancies in $\mathrm{GaN},{ }^{29}$ manifesting the radiation hardness of $\mathrm{ZnO}$. In addition, as the introduction rates of primary defects are typically 
$\simeq 1 \mathrm{~cm}^{-1}$, both the $\mathrm{Zn}$ vacancies and negative ions are probably formed through recombination processes during the irradiation and are thus parts of defect complexes. This is supported by the observations that the isolated $\mathrm{Zn}$ vacancies ${ }^{6}$ and probably isolated interstitials on both sublattices ${ }^{12}$ are mobile well below room temperature. On the other hand, the introduction rate of the $\mathrm{O}$ vacancies suggests that they are primary defects, and comparison to results obtained from EPR measurements, ${ }^{11,13}$ which show that the isolated $\mathrm{O}$ vacancy is stable at room temperature, suggests that the $\mathrm{O}$ vacancies observed in this work are isolated.

\section{Summary}

We observe three kinds of point defects produced by the electron irradiation in $n$-type $\mathrm{ZnO}$ : vacancies on both sublattices (possibly parts of larger defect complexes) and negative-ion-type defects, attributed to $\mathrm{O}$ interstitials or $\mathrm{O}$ antisites. The $\mathrm{Zn}$ vacancies and the negative ions act as compensating centers, both produced at concentrations $\left[V_{\mathrm{Zn}}\right]$ $\simeq c_{\mathrm{st}} \simeq 2 \times 10^{16} \mathrm{~cm}^{-3}$ and are probably parts of larger defect complexes. The irradiation-induced $\mathrm{O}$ vacancies are suggested to be isolated and have a deep donor character with the ionization level $E_{i}=100 \pm 10 \mathrm{meV}$ below the conduction band. They are produced at a concentration of $\left[V_{\mathrm{O}}\right] \simeq 3$ $\times 10^{17} \mathrm{~cm}^{-3}$. The lifetime of a positron trapped at an $\mathrm{O}$ vacancy is determined to be $\tau_{V, O}=195 \pm 5 \mathrm{ps}$, while it is $\tau_{V, \mathrm{Zn}}$ $=230 \pm 10 \mathrm{ps}$ in the $\mathrm{Zn}$ vacancy. ${ }^{21}$

\section{THERMAL RECOVERY}

\section{A. Positron data}

Both the as-grown and irradiated $\mathrm{ZnO}$ samples were in situ thermally annealed in the positron measurement cryostat for $30 \mathrm{~min}$ at temperatures up to $600 \mathrm{~K}$. No changes in the positron parameters were observed in as-grown $\mathrm{ZnO}$. The average positron lifetime measured at four different temperatures in the irradiated samples is presented as a function of annealing temperature in Fig. 6. The positron lifetime decreases with increasing annealing temperature and reaches the level of the as-grown material at the annealing temperature of $590 \mathrm{~K}$, above which no further changes were observed. The two lifetime components $\tau_{1}=150-180 \mathrm{ps}$ and $\tau_{2}=230 \pm 10 \mathrm{ps}$ extracted from the data are also shown in Fig. 6 together with the intensity of the second component. The experimental spectra are two-componential up to the highest annealing temperatures, where the separation could be performed only at low measurement temperatures due to the small concentration of the defects.

As can be seen from Fig. 6, the first annealing stage of the irradiation-induced defects occurs at temperatures around $400 \mathrm{~K}$. Figure 7 presents the average positron lifetime as a function of measurement temperature after the annealing steps at $400-430 \mathrm{~K}$. The average positron lifetime decreases by $3-5 \mathrm{ps}$ at all measurement temperatures during this annealing stage. The intensity of the $\mathrm{Zn}$ vacancy-related lifetime component $\tau_{2}=230 \mathrm{ps}$ decreases at this stage (upmost panel in Fig. 6), while the component itself remains constant,

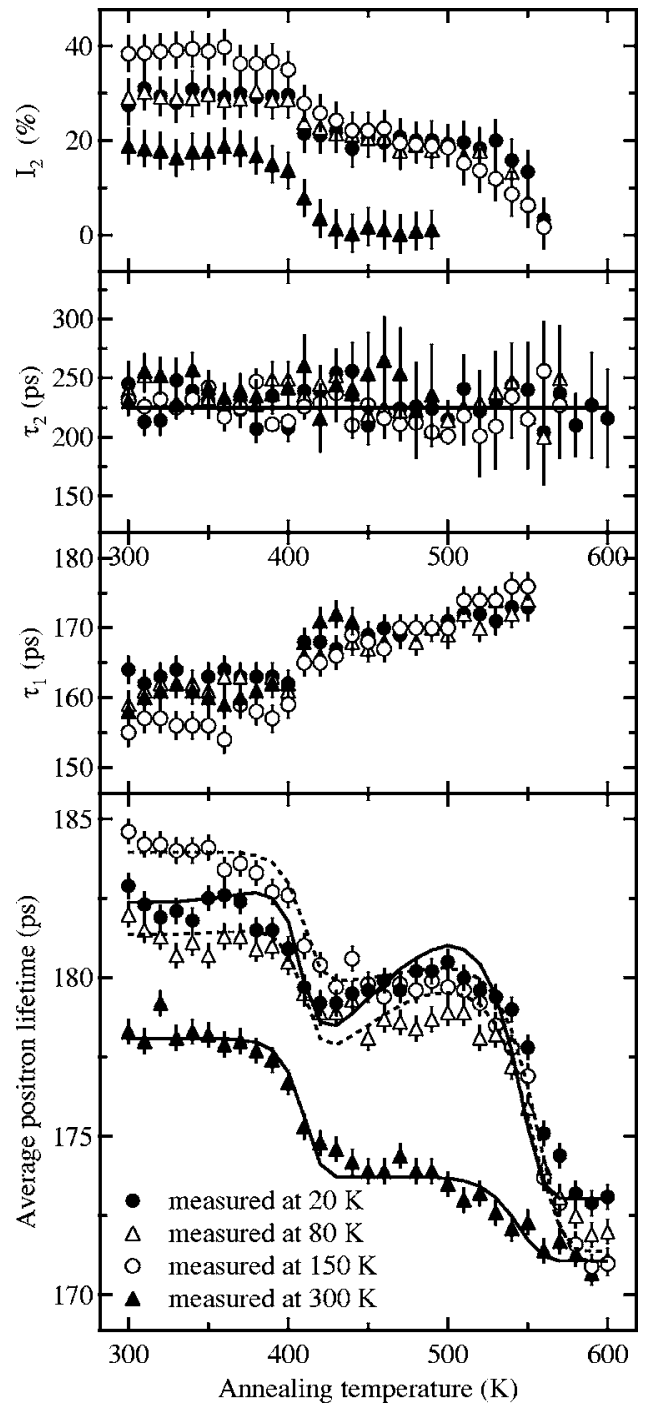

FIG. 6. The average positron lifetime in the irradiated sample as a function of the annealing temperature, measured at 20, 80, 150, and $300 \mathrm{~K}$. The solid curves are obtained from the trapping rate fittings. The two extracted lifetime components are shown in the upper panels together with the intensity of the higher component.

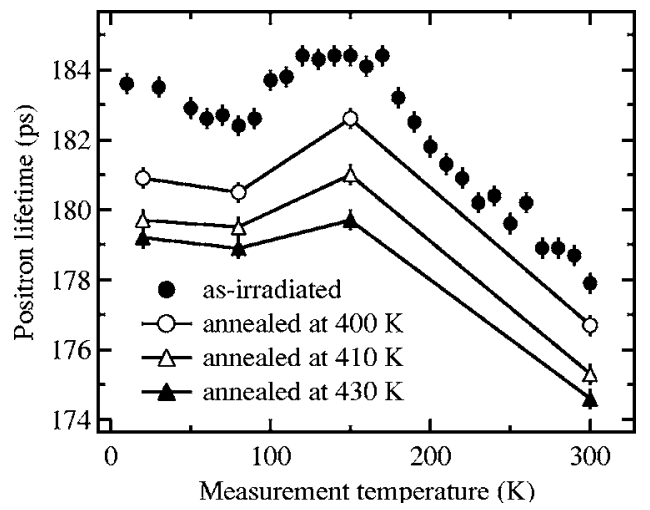

FIG. 7. The average positron lifetime in the irradiated sample annealed at temperatures $400-430 \mathrm{~K}$. 


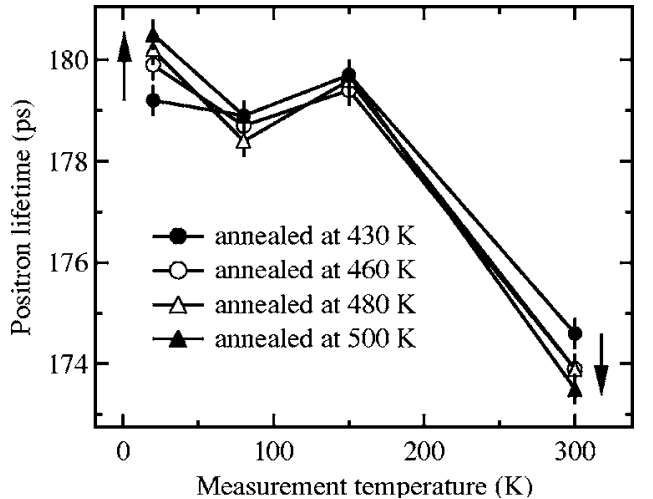

FIG. 8. The average positron lifetime in the irradiated sample annealed at temperatures $430-500 \mathrm{~K}$. The arrows show the direction of increasing annealing temperature.

indicating that part of the $\mathrm{Zn}$ vacancies anneal out of the material.

At annealing temperatures $430-500 \mathrm{~K}$ the average positron lifetime remains practically constant at measurement temperatures $80 \mathrm{~K}$ and $150 \mathrm{~K}$, increases at $20 \mathrm{~K}$, and decreases at $300 \mathrm{~K}$. This causes the $\tau_{\text {ave }}$ vs $T$ to become steeper (Fig. 8). The increase of the average positron lifetime at low temperatures can be explained by the annealing of the negative-ion-type defects which leads to a larger fraction of positron annihilations at $\mathrm{Zn}$ vacancies.

The full recovery of the irradiation-induced defects occurs at annealing temperatures $500-600 \mathrm{~K}$, as can be seen from Figs. 6 and 9. The local maximum in the $\tau_{\text {ave }}$ vs $T$ plot in Fig. 9 disappears at this last stage, which indicates that $\mathrm{O}$ vacancies anneal out simultaneously with the rest of the $\mathrm{Zn}$ vacancies. The positron lifetime versus measurement temperature curve in $\mathrm{ZnO}$ irradiated and annealed at $600 \mathrm{~K}$ is identical to that in as-grown $\mathrm{ZnO}$. This result is in perfect agreement with the results of electrical measurements, ${ }^{19}$ where the compensating defects produced by irradiation have been observed to anneal out of the material at around $600 \mathrm{~K}$.

The behavior of the separated lifetime components $\tau_{1}$ and $\tau_{2}$ as a function of annealing temperature (Fig. 6) gives fur-

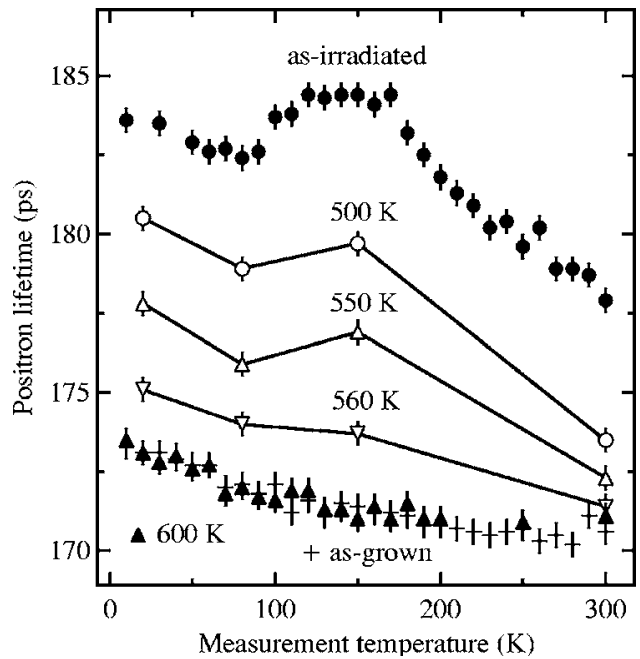

FIG. 9. The average positron lifetime in the irradiated sample annealed at temperatures 500-600 K.

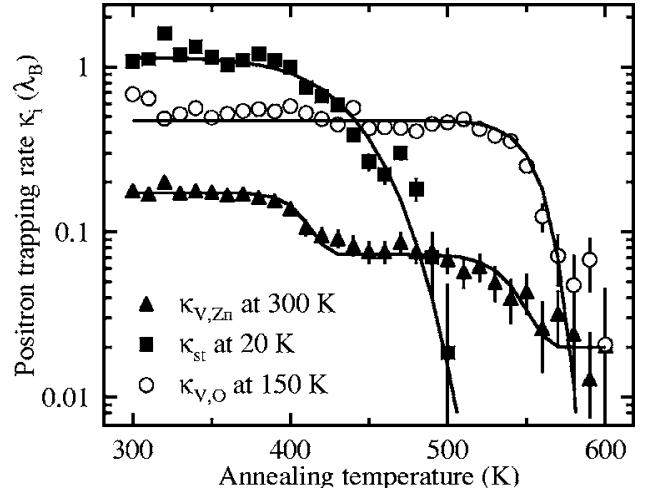

FIG. 10. The positron trapping rates to the different defects as a function of the annealing temperature. The solid curves are fitted to the data.

ther support for the three-defect model presented in Sec. III. The second lifetime component $\tau_{2}$, specific to the $\mathrm{Zn}$ vacancy, remains constant throughout the whole annealing temperature range, implying that it truly represents positrons annihilating in one bound state rather than a superposition of several bound states. The first (lower) lifetime component $\tau_{1}$ increases abruptly at the first annealing stage, as expected since the trapping rate to the $\mathrm{Zn}$ vacancies decreases due to the decrease in their concentration. However, the experimental value of $\tau_{1}$ after this stage coincides with the bulk lifetime $\left(\tau_{1}=\tau_{B}=170 \mathrm{ps}\right)$, while it should be lower in the ideal case of positron trapping at $\mathrm{Zn}$ vacancies only. ${ }^{23} \mathrm{In}$ addition, $\tau_{1}$ increases even above $\tau_{B}$ with further annealing, giving a direct indication that it is mixed with a lifetime component clearly above $\tau_{B}$. This mixing implies the presence and domination of an additional vacancy defect after the annealing at $500 \mathrm{~K}$, although it is not possible to decompose directly more than two lifetime components due to finite statistics. This vacancy defect is the O vacancy, identified in Sec. III by Doppler broadening measurements.

\section{B. Analysis}

It was shown in Sec. III B that the trapping rates to the $\mathrm{Zn}$ vacancies, negative-ion-type defects, and $\mathrm{O}$ vacancies can be directly estimated from the positron data without fitting. Using Eqs. (7)-(9) the trapping rates to the three defects were calculated as a function of annealing temperature. They are presented in Fig. 10. It is clearly seen that the interpretation of the results presented above is correct: the $\mathrm{Zn}$ vacancies anneal out of the material at two separate stages at around $400 \mathrm{~K}$ and $550 \mathrm{~K}$, the negative-ion-type defects anneal out around $450 \mathrm{~K}$, and the $\mathrm{O}$ vacancies anneal out together with the second part of the $\mathrm{Zn}$ vacancies above $550 \mathrm{~K}$. The temperature at which the $\mathrm{O}$ vacancies recover is in good agreement with a recent EPR study, ${ }^{13}$ where the $\mathrm{O}$ vacancies are observed to disappear between $570 \mathrm{~K}$ and $670 \mathrm{~K}$.

The activation energies $E_{A}$ corresponding to the different annealing stages can be fitted to the positron data. Assuming that each defect anneals independently, the isochronal annealing process for a defect concentration $N$ can be described as $^{19}$ 


$$
N_{i+1}=N_{\infty}+\left(N_{i}-N_{\infty}\right) \exp \left[-\nu t \exp \left(-E_{A} / k T_{i}\right)\right],
$$

where the subscript $i$ denotes the annealing step $\left(T_{i}=[300\right.$ $+10 \times(i-1)] \mathrm{K}), t=1800 \mathrm{~s}$ is the annealing time, and $\nu$ is a frequency factor (assumed to be $\nu=10^{13} \mathrm{~s}^{-1}$ ). The trapping rates are directly proportional to the defect concentrations. Thus the trapping rates and activation energies of the annealing stages can be fitted to the data in Fig. 10.

Using the above model, two separate activation energies for the $\mathrm{Zn}$ vacancies were fitted, giving $E_{A 1}^{V, \mathrm{Zn}}=1.3 \pm 0.1 \mathrm{eV}$ and $E_{A 2}^{V, Z n}=1.8 \pm 0.1 \mathrm{eV}$. Here $40 \%$ of the irradiation-induced $\mathrm{Zn}$ vacancies recover at the first anneling stage. The activation energy of the second stage is in perfect agreement with the value obtained in the electrical measurements for the annealing of the compensating center, $E_{A}=1.73 \mathrm{eV} .{ }^{19}$ The activation energy of the annealing stage of the $\mathrm{O}$ vacancies is fitted as $E_{A}^{V, O}=1.8 \pm 0.1 \mathrm{eV}$, which coincides with that of the second stage of the $\mathrm{Zn}$ vacancies, as expected.

The recovery of the negative-ion-type defects could not be fitted with a single activation energy. Instead, a linear dependence $E_{A}^{\mathrm{st}}=E_{A 0}^{\mathrm{st}}+\alpha\left(T_{i}-T_{1}\right)$ was assumed. The fitting gave $E_{A 0}^{\mathrm{st}}=1.15 \mathrm{eV}$ and $\alpha=0.0023$, with the activation energies ranging from $1.4 \pm 0.1 \mathrm{eV}$ at the beginning of the anneal at about $400 \mathrm{~K}$ to $1.6 \pm 0.1 \mathrm{eV}$ at the end at about $500 \mathrm{~K}$. This kind of "sliding" of the activation energy can be expected if the negative-ion-type defects are not all identical and have different diffusion barriers. Another possibility would be that the out-annealing of these defects is not limited only by diffusion, but, e.g., by the transition to the surface or to another lattice site.

The low introduction rate of the $\mathrm{Zn}$ vacancies suggests that they have formed complexes with other defects. In addition, already the early EPR studies ${ }^{6}$ have shown that the isolated $\mathrm{Zn}$ vacancy is mobile well below room temperature. The fact that only the irradiation-induced $\mathrm{Zn}$ vacancies have annealed out of the material at $600 \mathrm{~K}$ implies that the ingrown $\mathrm{Zn}$ vacancies are not isolated (as expected, since they have survived the cooling down from the growth temperatures) and that the complexes formed during the irradiation are different from the in-grown Zn-vacancy-related defect. In addition, the observation of two separate annealing stages of the $\mathrm{Zn}$ vacancies indicates that two different defect complexes are formed in the irradiation.

\section{Summary}

We observe three annealing stages of the point defects produced by electron irradiation in $n$-type $\mathrm{ZnO}$ in the temperature range $300-600 \mathrm{~K}$. The first annealing stage occurs at about $400 \mathrm{~K}$ and consists of the recovery of $40 \%$ of the irradiation-induced $\mathrm{Zn}$ vacancies, with an activation energy of $E_{A 1}^{V, \mathrm{Zn}}=1.3 \pm 0.1 \mathrm{eV}$. The second annealing stage covers the temperature range $400-500 \mathrm{~K}$, where the negative-ion-type defects recover, with activation energies ranging from $1.4 \pm 0.1 \mathrm{eV}$ at the beginning of the anneal at about $400 \mathrm{~K}$ to $1.6 \pm 0.1 \mathrm{eV}$ at the end at about $500 \mathrm{~K}$. The third annealing stage occurs at about $550 \mathrm{~K}$ and consists of the recovery of the $\mathrm{O}$ vacancies and of the remaining irradation-induced $\mathrm{Zn}$ vacancies, with an activation energy of $E_{A 2}^{V, Z n} \simeq E_{A}^{V, O}$

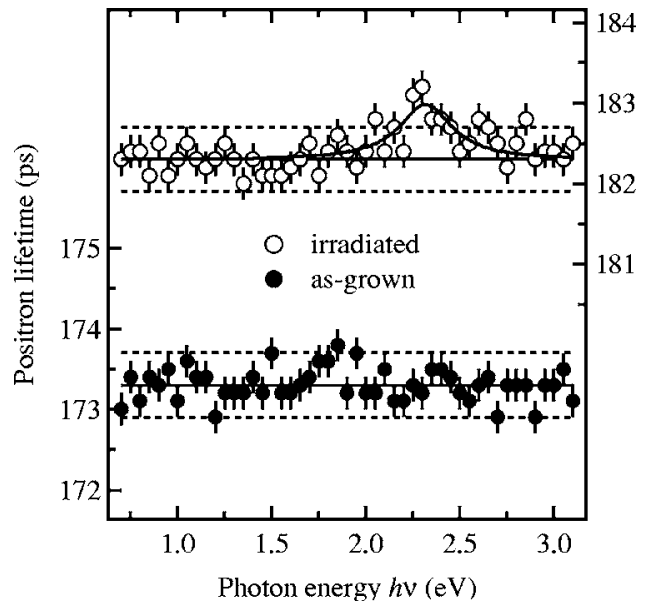

FIG. 11. The average positron lifetime at $20 \mathrm{~K}$ in the as-grown and irradiated $\mathrm{ZnO}$ samples as a function of illumination photon energy. The solid and dashed lines show the corresponding average lifetimes in the dark \pm 2 standard deviations. The solid curve is drawn to guide the eye.

$=1.8 \pm 0.1 \mathrm{eV}$. The positron data show that the irradiationinduced defects have fully recovered after this final annealing stage, in perfect agreement with the results of electrical measurements. ${ }^{19}$

\section{ILLUMINATION EXPERIMENTS}

We studied the optical activity of the in-grown and irradiation-induced defects by shining monochromatic light on the samples during the positron experiments at $20 \mathrm{~K}$. The results are presented in Fig. 11. No effect is observed in the as-grown $\mathrm{ZnO}$ samples, where the average positron lifetime remains constant when the photon energy is varied between 0.7 and $3.1 \mathrm{eV}$. On the other hand, in the irradiated material, a small increase of the average positron lifetime is visible when the photon energy is increased to $2.3 \mathrm{eV}$, followed by a decrease to the value in the dark with further increase of the photon energy.

Due to limitations imposed by the illumination equipment, the photon flux decreased above $2.4 \mathrm{eV}$. In order to confirm that the decrease in the average positron lifetime above $2.3 \mathrm{eV}$ is not due this decrease in the photon flux, we performed measurements with different photon fluxes at 2.3 eV. As can be seen from Fig. 12, the average positron lifetime increases up to a photon flux of $7 \times 10^{14} \mathrm{~cm}^{-2} \mathrm{~s}^{-1}$. The average lifetime stays at the same increased level when the flux is further increased to $6 \times 10^{15} \mathrm{~cm}^{-2} \mathrm{~s}^{-1}$ with the help of 540-nm lasers, which shows that the optically active electrons have reached an equilibrium already at the flux of 7 $\times 10^{14} \mathrm{~cm}^{-2} \mathrm{~s}^{-1}$. Thus the increase of the positron lifetime at photon energy $2.3 \mathrm{eV}$ in Fig. 11 is not limited by the applied photon flux and the behavior of the positron lifetime as a function of illumination photon energy in the irradiated samples is caused by two separate optical processes.

The behavior of the average positron lifetime under illumination at low temperature in Fig. 11 can be explained in the following way. As seen from Fig. 5, the positron data are 


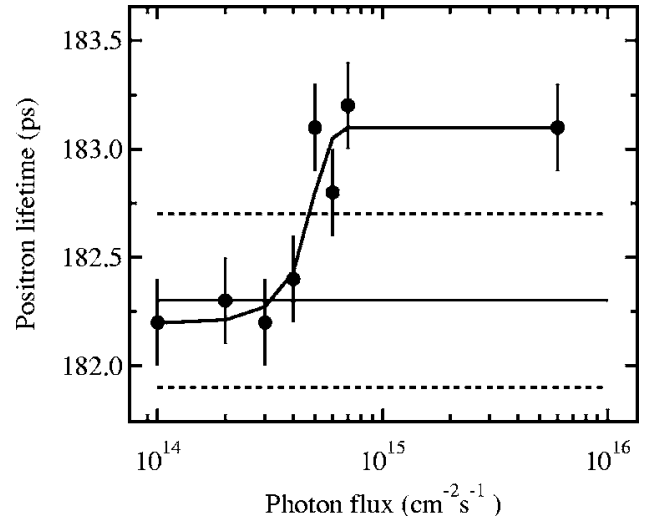

FIG. 12. The average positron lifetime at $20 \mathrm{~K}$ in the irradiated $\mathrm{ZnO}$ samples as a function of illumination photon $(E=2.3 \mathrm{eV})$ flux. The solid and dashed lines show the corresponding average lifetimes in the dark \pm 2 standard deviations. The solid curve is drawn to guide the eye.

dominated by the $\mathrm{Zn}$ vacancies and negative-ion-type defects at the measurement temperature of $20 \mathrm{~K}$. Thus it is natural to assign the observed optical activity to these defects. The $\mathrm{Zn}$ vacancies are mostly in the double-negative-charge state ${ }^{21}$ (and cannot become more negative), and hence the increase of the average positron lifetime when approaching the illumination photon energy of $2.3 \mathrm{eV}$ from below indicates that the negative-ion-type defects become less negative or even neutral and start trapping positrons less efficiently, increasing the fraction of positron annihilations at the $\mathrm{Zn}$ vacancies. The subsequent decrease of the positron lifetime with the further increase of the illumination photon energy then indicates that the $\mathrm{Zn}$ vacancies become less negative in their turn. This can be interpreted as both the $\mathrm{Zn}$ vacancies and the negative ions having an ionization level close to $2.3 \mathrm{eV}$. According to theoretical calculations, ${ }^{15}$ the $V_{\mathrm{Zn}}^{2-/-}$ transition should occur at $E_{C}-2.6 \mathrm{eV}$, in good agreement with our data. The same work predicts that the $\mathrm{O}$ interstitial (octahedral position) has the $\mathrm{O}_{I}^{2-/}$ transition at $E_{C}-2.4 \mathrm{eV}$ and the $\mathrm{O}$ antisite has the $\mathrm{O}_{\mathrm{Zn}}^{2-/ 0}$ transition at $E_{C}-2.3 \mathrm{eV}$. This supports the tentative identification of the negative ion as the $\mathrm{O}_{I}$ or $\mathrm{O}_{\mathrm{Zn}}$.

All the optical activity is lost after the thermal annealings, and the $\tau_{\text {ave }}$ versus photon energy of the irradiated samples annealed at $600 \mathrm{~K}$ coincides with that of the as-grown samples. The free-electron concentration in both the asgrown and irradiated and annealed $\mathrm{ZnO}$ is 30 times larger than after the irradiation. Thus, even if there were an optical transition at around $2.3 \mathrm{eV}$ in the as-grown material, it would probably not be observed with the photon fluxes used in this work since the recombination rate is higher due to free electrons. The electron level at $2.3 \mathrm{eV}$ below the conduction band correlates with the energy of the emitted light in green luminescence. ${ }^{14}$ Thus the $\mathrm{Zn}$ vacancies, which have the lowest defect formation energy in $n$-type $\mathrm{ZnO},{ }^{15}$ could be involved in the transition giving rise to the green luminescence.

\section{CONCLUSIONS}

We have applied positron annihilation spectroscopy to study the point defects introduced by $2-\mathrm{MeV}$ electron irradiation at room temperature in $n$-type $\mathrm{ZnO}$. The measurements were performed at $10-300 \mathrm{~K}$. We studied the optical activity of the irradiation-induced defects by shining monochromatic light on the samples during the positron measurements at $20 \mathrm{~K}$. The thermal recovery of the defects produced by the irradiation was studied by 30 -min annealings of the samples at 300-600 K.

We observe three kinds of point defects produced by the electron irradiation: vacancies on both sublattices and negative-ion-type defects, tentatively identified as $\mathrm{O}$ interstitials. The $\mathrm{Zn}$ vacancies and the negative ions act as compensating centers, both produced at concentrations $\left[V_{\mathrm{Zn}}\right] \simeq c_{\mathrm{st}}$ $\simeq 2 \times 10^{16} \mathrm{~cm}^{-3}$. The irradiation-induced $\mathrm{O}$ vacancies are deep donors with the ionization level about $100 \mathrm{meV}$ below the conduction band and produced at a concentration of $\left[V_{\mathrm{O}}\right] \simeq 3 \times 10^{17} \mathrm{~cm}^{-3}$. The lifetime of a positron trapped at an $\mathrm{O}$ vacancy is determined to be $\tau_{V, O}=195 \pm 5 \mathrm{ps}$, while it is $\tau_{V, Z n}=230 \pm 10 \mathrm{ps}$ in the $\mathrm{Zn}$ vacancy.

The irradiation-induced defects fully recover after the annealing at $600 \mathrm{~K}$, in good agreement with electrical measurements. The $\mathrm{Zn}$ vacancies anneal out of the material in two stages with fitted activation energies $E_{A 1}^{V, Z n} \simeq 1.3 \mathrm{eV}$ and $E_{A 2}^{V, Z n} \simeq 1.8 \mathrm{eV}$, indicating that the $\mathrm{Zn}$ vacancies are parts of two different defect complexes. The $\mathrm{O}$ vacancies anneal simultaneously with the $\mathrm{Zn}$ vacancies at the later stage with fitted activation energy $E_{A}^{V, O} \simeq 1.8 \mathrm{eV}$. The negative-ion-type defects anneal out between the two annealing stages of the $\mathrm{Zn}$ and $\mathrm{O}$ vacancies. No single activation energy could be fitted to this annealing stage, indicating that the recovery of these defects is not limited only by the diffusion in the lattice.

By shining monochromatic light on the samples during the positron annihilation measurements at low temperature, we find that both the irradiation-induced $\mathrm{Zn}$ vacancies and the negative-ion-type defects have ionization levels close to $2.3 \mathrm{eV}$. Based on this we suggest that the negative ions are oxygen interstitials or oxygen antisites in the negative-charge state (similarly to the $\mathrm{Zn}$ vacancies). No optical activity was observed in the as-grown $\mathrm{ZnO}$ or in the irradiated $\mathrm{ZnO}$ after annealing at $600 \mathrm{~K}$. Since the $\mathrm{Zn}$ vacancies have the lowest defect formation energy in $n$-type $\mathrm{ZnO}$, it is likely that they are involved in the transition responsible for the green luminescence in $\mathrm{ZnO}$.

\section{ACKNOWLEDGMENTS}

We wish to thank G. Cantwell for providing the samples. F.T. acknowledges financial support from Emil Aaltonen and Jenny and Antti Wihuri foundations and from the Research Foundation of Helsinki University of Technology. D.C.L. was supported by U.S. Air Force Contract No. F33615-00C-5402. 
*Electronic address: filip.tuomisto@tkk.fi

${ }^{1}$ D. C. Look, D. C. Reynolds, J. R. Sizelove, R. L. Jones, C. W. Litton, G. Cantwell, and W. C. Harsch, Solid State Commun. 105, 399 (1998).

${ }^{2}$ K. K. Kim, H. S. Kim, D. K. Hwang, J. H. Lim, and S. J. Park, Appl. Phys. Lett. 83, 63 (2003).

${ }^{3}$ Y. R. Ryu, T. S. Lee, and H. W. White, Appl. Phys. Lett. 83, 87 (2003).

${ }^{4}$ D. C. Look, G. M. Renlund, R. H. Burgener II, and J. R. Sizelove, Appl. Phys. Lett. 85, 5269 (2004).

${ }^{5}$ P. Sharma, A. Gupta, K. V. Rao, F. J. Owens, R. Sharma, R. Ahuja, J. M. Osorio Guillen, B. Johansson, and G. A. Gehring, Nat. Mater. 2, 673 (2003).

${ }^{6}$ D. Galland and A. Herve, Solid State Commun. 14, 953 (1974).

${ }^{7}$ D. Galland and A. Herve, Phys. Lett. 33A, 1 (1970).

${ }^{8}$ K. Leutwein and J. Schneider, Z. Naturforsch. A 26, 1236 (1971).

${ }^{9}$ A. L. Taylor, G. Filipovich, and G. K. Lindeberg, Solid State Commun. 8, 1359 (1970).

${ }^{10}$ J. M. Smith and W. E. Vehse, Phys. Lett. 31A, 147 (1970).

${ }^{11}$ C. Gonzales, D. Galland, and A. Herve, Phys. Status Solidi B 72, 309 (1975).

${ }^{12}$ Y. V. Gorelkinskii and G. D. Watkins, Phys. Rev. B 69, 115212 (2004).

${ }^{13}$ L. S. Vlasenko and G. D. Watkins, Phys. Rev. B 71, 125210 (2005).

${ }^{14}$ D. C. Reynolds, D. C. Look, B. Jogai, and H. Morkoc, Solid State Commun. 101, 643 (1997)

${ }^{15}$ A. F. Kohan, G. Ceder, D. Morgan, and Chris G. Van de Walle, Phys. Rev. B 61, 15019 (2000).

${ }^{16}$ K. Vanheusden, C. H. Seager, W. L. Warren, D. R. Tallant, and J.
A. Voight, Appl. Phys. Lett. 68, 403 (1995).

${ }^{17}$ K. Vanheusden, W. L. Warren, C. H. Seager, D. R. Tallant, J. A. Voight, and B. E. Gnade, J. Appl. Phys. 79, 7983 (1996).

${ }^{18}$ H.-J. Egelhaaf and D. Oelkrug, J. Cryst. Growth 161, 190 (1996).

${ }^{19}$ D. C. Look, D. C. Reynolds, J. W. Hemsky, R. L. Jones, and J. R. Sizelove, Appl. Phys. Lett. 75, 811 (1999).

${ }^{20}$ A. Y. Polyakov et al., J. Appl. Phys. 94, 2895 (2003).

${ }^{21}$ F. Tuomisto, V. Ranki, K. Saarinen, and D. C. Look, Phys. Rev. Lett. 91, 205502 (2003).

${ }^{22}$ C. Coskun, D. C. Look, G. C. Farlow, and J. R. Sizelove, Semicond. Sci. Technol. 19, 752 (2004).

${ }^{23}$ K. Saarinen, P. Hautojärvi, and C. Corbel, in Identification of Defects in Semiconductors, edited by M. Stavola (Academic Press, New York, 1998), p. 209.

${ }^{24}$ K. Saarinen, A. P. Seitsonen, P. Hautojärvi, and C. Corbel, Phys. Rev. B 52, 10932 (1995).

${ }^{25}$ C. Corbel, F. Pierre, K. Saarinen, P. Hautojärvi, and P. Moser, Phys. Rev. B 45, 3386 (1992).

${ }^{26}$ M. J. Puska, C. Corbel, and R. M. Nieminen, Phys. Rev. B 41, 9980 (1990).

${ }^{27}$ J. Oila, J. Kivioja, V. Ranki, K. Saarinen, D. C. Look, R. J. Molnar, S. S. Park, S. K. Lee, and J. Y. Han, Appl. Phys. Lett. 82, 3433 (2003).

${ }^{28}$ K. Saarinen, J. Nissilä, P. Hautojärvi, J. Likonen, T. Suski, I. Grzegory, B. Lucznik, and S. Porowski, Appl. Phys. Lett. 75, 2441 (1999).

${ }^{29}$ K. Saarinen, T. Suski, I. Grzegory, and D. C. Look, Phys. Rev. B 64, 233201 (2001).

${ }^{30}$ J. Mäkinen, C. Corbel, P. Hautojärvi, P. Moser, and F. Pierre, Phys. Rev. B 39, 10162 (1989). 